\title{
Space operation system for Chang'E program and its capability evaluation
}

\author{
Yu Zhi-Jian, Lu Li-Chang, Liu Yung-Chun and Dong Guang-Liang \\ Beijing Institute of Tracking and Telecommunications Technology, P.O. Box 5131, Beijing 100 094, China. \\ *e-mail: csaspace@163bj.com
}

Space operation for China's first lunar exploration program, Chang'E will be provided by the S-band aerospace Telemetry, Tracking and Command (TT\&C) network designed for China's manned space program. This is undoubtedly a great challenge to the ground TT\&C system. The largest antennas of China's S-band aerospace TT\&C network has an aperture of only $12 \mathrm{~m}$. A series of technical measures have been taken into the designing of the spacecraft-ground TT\&C system to ensure that such antennas can communicate with Chang'E-1 lunar probe 400,000 km away. These include installation of high-gain directional antennae and medium-gain omni-directional antennae for the probe, adding channel encoding to the downlink channel, using both high and low data rates for information transmission and upgrade and design of ground equipment terminals. Among them, the omni-directional antenna will operate in the earth-ground transfer orbit phase and the directional antenna will operate in the lunar orbit phase. These measures satisfy the spacecraftground link and program design requirements.

To provide accurate navigation for the probe during its Earth-Moon flight and initial lunar orbiting flight, China's VLBI system designed for astronomical observations, will also be used besides the ranging and range rate measurement capabilities of the S-band TT\&C network. The purpose is to provide $100 \mathrm{~m}$ accuracy in position determination during lunar orbit. This paper describes the system design, technical challenges, solutions and capability evaluation of space operation for Chang'E-1.

\section{Basic configuration of the space operation system for Chang'E program}

China will implement its lunar exploration program in the next few years. The program encompasses five major systems: launch vehicle, spacecraft, launch site, application system and TT\&C system. Among these, the main tasks of the TT\&C system will be tracking of the spacecraft through ground operation, data reception and space operation control. Large-aperture deep space TT\&C facilities are usually built to provide TT\&C support for lunar exploration programs in the world. However, such facilities require heavy investment. Due to the constraint in program cost, space operation for China's first lunar exploration program will be provided by the aerospace TT\&C network designed for China's manned space program. The TT\&C network consists of a flight control center and a number of S-band TT\&C stations and has already successfully provided reliable TT\&C support service for many domestic and foreign spacecrafts and for five spaceships in flight experiments of China's manned space program. All technical specifications of the network have been proven to meet the design requirements.

To provide tracking and instrumentation for Chang'E-1 lunar probe at a long range, a series of advance technologies have been incorporated in the TT\&C system, so that the $12 \mathrm{~m}$ S-band stations can cooperate with onboard equipment and support the mission at the available signal level.

Keywords. Chang'E program; lunar exploration; space operations; TT\&C design. 


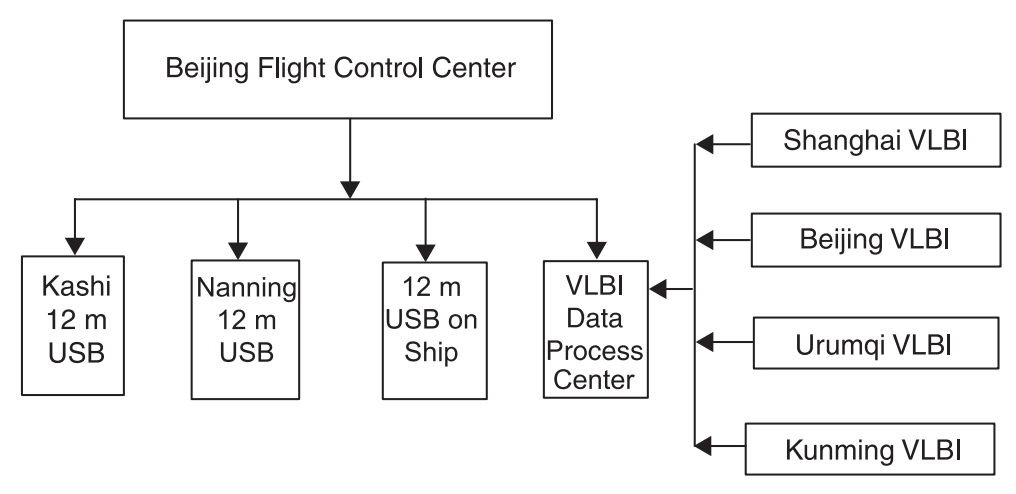

Figure 1. The Telemetry, Tracking and Command System for Chang'E program.

In addition to the use of the ranging and range rate capabilities of the S-band TT\&C network, the VLBI system made for astronomical observations in China will be used to provide initial accurate navigation for the Earth-Moon flight and moonorbiting phase of Chang'E-1 probe. Use of the VLBI system is also aimed at achieving an accuracy of $100 \mathrm{~m}$ for orbit determination during the lunar orbiting phase as required for accomplishing scientific objectives.

China completed its international standard S-band aerospace TT\&C network in 1998 to provide support for its manned space missions. The network consists of a network control center and three S-band TT\&C stations, having the largest antenna aperture of $12 \mathrm{~m}$. Besides, international cooperation is also sought with some foreign S-band TT\&C stations to provide support for this mission.

The $12 \mathrm{~m}$ S-band TT\&C systems are located in Kashi, Nanning and Yuanwang instrumentation ships. China's VLBI measurement system has four observation stations located in Shanghai, Beijing, Urumqi and Kunming. Their observation data can be transmitted in real time to the data processing center in Shanghai for processing, and the processing result can be sent to Beijing Flight Control Center, which is responsible for command, control and data processing for the Chang'E program.

\section{Design of the space-ground TT\&C link}

\subsection{Computation of the TTEC link margin}

The main technical specifications of the $12 \mathrm{~m}$ USB (Unified S-Band) stations are as follows:

- System uplink EIRP: $71 \mathrm{dBW}$

- Ground station system G/T: $22.5 \mathrm{~dB} / \mathrm{K}$

- Measurement accuracy:
Angular: $\quad S /\left.\Phi\right|_{C}=43 \mathrm{dBHz} ; \quad \sigma_{A E}=0.01^{\circ}$, $\Delta_{A E}=0.02^{\circ}$
Ranging: $S /\left.\Phi\right|_{\text {Major tone }}=35 \mathrm{dBHz} ; \sigma_{R}=13 \mathrm{~m}$, $\Delta_{R}=8 \mathrm{~m}$
Range rate: $S /\left.\Phi\right|_{C}=43 \mathrm{dBHz} ; \sigma=0.05 \mathrm{~m} / \mathrm{s}$

- TM bit rate 0.1 to $\sim 64 \mathrm{kbps}$, modulation PCMPSK/ PCM-DPSK

- TC bit rate 0.1 to $\sim 8 \mathrm{kbps}$ modulation PCMPSK

- Data signal bit rate $2 \mathrm{Mbps}$, modulation PCM/ QPSK

$\mathrm{G} / \mathrm{T}$ of China's $12 \mathrm{~m}$ antenna systems is $22.5 \mathrm{~dB} / \mathrm{K}$ and the EIRP is $71 \mathrm{dBW}$.

The equipment onboard Chang'E-1 probe has a $\mathrm{G} / \mathrm{T}$ of $-30 \mathrm{~dB} / \mathrm{K}$, EIRP $13 \mathrm{dBW}$

- The effective space-ground range is $400,000 \mathrm{~km}$

The omni-directional antenna operates in the high-gain zone (antenna gain: $0 \mathrm{dBi}$; EIRP: $13 \mathrm{dBW}$ ). Besides, atmospheric attenuation, polarization loss, space noise, etc. have to be taken into consideration. Telecommand and telemetry bit rate for lunar spacecraft is usually low for a lunar exploration mission. Bit rate of telemetry usually does not exceed $1 \mathrm{kbps}$; we use this value for telemetry. Bit rate for the telecommand is $250 \mathrm{bps}$. Based on preliminary analysis, the S-band TT\&C stations should be able to meet the requirements of the lunar orbiter program after appropriate adaptive modifications. Directional antenna operation mode is used for downlinking. Because the EIRP of the directional antenna is $5 \mathrm{~dB}$ greater than the high-gain zone of the omni-direction antenna, $5 \mathrm{~dB}$ margin is added to the downlink signal.

\subsection{Results of computation}

Our calculations show that the requirement on $\mathrm{G} / \mathrm{T}$ of the spacecraft-borne equipment is not high, and mission requirements will be met with $-36 \mathrm{~dB} / \mathrm{K}$. However, the downlink has a high requirement of over $13 \mathrm{dBW}$ on EIRP 
Table 1. Calculation of margins of the TTEC channels (dB).

\begin{tabular}{lcccc}
\hline & $\begin{array}{c}\text { Mode 1 } \\
\text { Telemetry only }\end{array}$ & $\begin{array}{c}\text { Mode 2 } \\
\text { Telecommand, telemetry } \\
\text { and ranging }\end{array}$ & $\begin{array}{c}\text { Mode 3 } \\
\text { Ranging acquisition } \\
\text { and telemetry }\end{array}$ & $\begin{array}{c}\text { Mode } 4 \\
\text { Telecommand and } \\
\text { telemetry }\end{array}$ \\
\hline TC & - & 14 & - & 17 \\
TM & 8 & 6 & 6 & 8 \\
Major tone & - & 5 & 5 & - \\
Range rate & 8 & 7 & 7 & 8 \\
\hline
\end{tabular}

of the spacecraft-borne antenna. Therefore, the spacecraft-borne transmitter should have a power of $20 \mathrm{~W}$. As a result, a high requirement is imposed on the spacecraft-borne antenna pattern. Besides, near-omni-directional earth coverage is required from the spacecraft-borne antenna because the lunar probe will undergo significant changes of attitude during its flight and, at the same time, TT\&C of the spacecraft should still be possible when the attitude of the spacecraft is not stable. It is a challenging task for an omni-directional antenna to attain such high performance.

Multiple operation modes can be used by a unified carrier S-band (USB) system, and these include:

Mode 1: Telemetry only

Mode 2: Telecommand, telemetry and ranging

Mode 3: Ranging acquisition and telemetry

Mode 4: Telecommand and telemetry

The table 1 gives margins of system level for different operation modes.

\subsection{Technical challenges}

It is a great challenge to use the existing S-band TT\&C network to provide support for the lunar spacecraft. To meet this challenge, we have to take measures in the designing of the spacecraft-borne and ground TT\&C systems to increase their technical capabilities. These measures include:

- Layout of the transponders and antennas should be optimized to reduce loss of feedlines;

- Allocation of signal energy can be optimized through an appropriate adjustment of uplink and downlink modulation.

- A range ambiguity-resolution technology combining software and hardware will be used.

Besides, Low Density Parity Check (LDPC) encoding technology can be used for reception of telemetry signals in designing the ground system.

LDPC code was proposed by R G Gallager in 1963 , but it was not possible to analyze and verify its performance through simulation because of the low level of development of computer technology and hardware. LDPC became a hot subject of research in 1995 when the Turbo code was put forth and received wide attention. Following that, M Luby et al made improvements on LDPC code and formed irregular LDPC code, that is, they changed the columns and rows of the original LDPC code parity rectangular matrix from single order to multiple orders so that LDPC acquires performance approaching Shannon limit. Its technical advantages are:

- Low decoding threshold: its encoding gain is far greater than convolution code. At an ideal condition of BER of $10^{-5}$, LDPC code has $2.8 \mathrm{~dB}$ more performance gain over $(2,1,7)$ convolution code.

- Having strong anti-burst error capability.

- Low decoding complexity.

Its delay is similar to that of convolution encoding/Viterbi decoding.

We have got the following conclusion based on testing on hardware:

At BER of $10^{-5}$ :

- $E_{b} / N_{0}=9.7 \mathrm{~dB}$ in case of non-encoding.

- When the demodulator outputs 3-bit soft decision information, $E_{b} / N_{0}=3.1 \mathrm{~dB}$ when LDPC code is used for coding and decoding, and $E_{b} / N_{0}=4.9 \mathrm{~dB}$ when convolution coding/ Viterbi decoding is used. LDPC coding and decoding is $1.8 \mathrm{~dB}$ higher than convolution coding/Viterbi decoding.

- Computer-aided instrumentation method assisted by channel information is used. When the demodulator outputs 3-bit soft decision information, $E_{b} / N_{0}=2.9 \mathrm{~dB}$ when LDPC code is used for coding and decoding. When the demodulator outputs 6-bit soft decision information, $E_{b} / N_{0}=2.2 \mathrm{~dB}$ when LDPC code is used for coding and decoding. LDPC coding and decoding is $2.7 \mathrm{~dB}$ higher than convolution coding/Viterbi decoding. 


\section{TT\&C operation modes}

\subsection{Operation mode of the probe-borne equipment}

As in operation mode of the spacecraft-borne equipments, the spacecraft should use omnidirectional antenna for reception as well as for transmission because of significant changes in the attitude of the spacecraft during its flight.

Besides, spacecraft-borne directional antenna is a key to deep space communication technology, high-data rate science data takes wide channel bandwidth, and it is no longer possible to receive the data with a non-directional antenna. A directional antenna can also be used for tracking and control of the spacecraft and can serve as backup. In an abnormal case when attitude of the spacecraft is not stable or the directional antenna fails, the ground will have to rely on the omni-directional antenna to provide emergency support.

\subsection{Operation mode of the ground TTEC network}

Beijing Flight Control Center organizes implementation of the TT\&C missions, carries out remote monitoring of the S-band stations including setting of operational parameters and monitoring of the operation status. The center will also provide accurate spacecraft orbit data and satellite telemetry parameter reports for operation and control of the spacecraft. Instrumentation data of the VLBI facilities are sent through data links to the flight control center and incorporated in orbit analysis and computation. VLBI instrumentation data are relatively independent of the S-band network and are only used for comprehensive data processing.

\section{Orbit measurement and orbit determination}

Deep space radio tracking relied solely on Doppler and range systems in the early deep space explorations. Though tracking of vehicles at lunar distances could be accomplished through a variety of radio and optical techniques, the radio communication provides the main tracking data. The navigation of Chang'E-1 will also rely on the range and Doppler data. However, two ground stations cannot take range and range-rate data simultaneously due to their limited capability. In addition, the radiometric tracking using S-band is done by single-frequency downlink. It is not possible to reduce the effects of the ionosphere and solar plasma. The orbit determination will mostly be based upon separated data observed by a single station.

To obtain preliminary estimates of such radio navigation capabilities, we have carried out simulation with the batch filtering technique of conventional radio data from stations considering the effects of error sources:

- Data coverage

- Length of data arc

- Constant station location errors

- Constant and stochastic spacecraft accelerations

- A priori knowledge of the lunar ephemeris

Simulation results indicate that navigation accuracy is associated with the geometry of the ground stations and the length of the tracking arc. Therefore the orbit determination capabilities could be enhanced with the availability of radio data tracked by stations located on sea.

In order to ensure the accuracy of the flight path of Chang'E-1 and provide navigation during its initial flight period around the Moon, the Chinese astronomical observation VLBI system is used besides USB, to measure the range and Doppler to accurately determine orbit. In the VLBI system, four antennas provide two approximately orthogonal baselines for satellite positioning. The evaluation of orbit determination capabilities of these two combined systems was also performed. The positional accuracy of Chang' $E$ in its lunar orbit is expected to be better than $100 \mathrm{~m}$.

\section{Evaluation of the comprehensive performance of the system}

When the omni-direction antenna is used onboard the spacecraft, the $12 \mathrm{~m}$ S-band ground TT\&C stations will be able to provide support for the spacecraft if the high-gain zone $(\mathrm{EIRP}=13 \mathrm{dBW})$ of the probe-borne omni-direction antenna covers the Earth. An omni-direction antenna has different degrees of low-gain zones (EIRP $=8 \mathrm{dBW})$. Based on link calculations, the ground system will have a small level margin in the low-gain zone earth coverage arcs. However, it can still provide TT\&C support for the spacecraft after appropriate measures are taken.

When the directional antenna is used onboard the spacecraft, the $12 \mathrm{~m}$ S-band ground stations will be able to provide the TT\&C support required for the spacecraft orbiting around the Moon and provide accurate measurement of the spacecraft range.

Using the ranging and range rate measurement information of the S-band TT\&C network and the VLBI system used for astronomical observations in China, the $100 \mathrm{~m}$ accuracy requirement 
of science targets for orbit determination during the lunar orbit phase could be met. Based on the above analysis, China will be able to use its own aerospace network and facilities to provide operation and control support for the lunar exploration spacecraft.
The use of existing deep space network facilities in the world to improve the reliability and support capabilities for the Chang'E program is also taken into consideration in the system design. Possible use of ESA's deep space stations in Spain and Perth is under consideration. 